\title{
Utilization of Lidar Technology to Assess Vertical Clearances of Civil Infrastructures
}

\author{
Levi McIntosh \\ Bashar Haddad \\ Mark Revels \\ Western Kentucky University \\ 1906 College Heights Blvd \\ Bowling Green, KY 42101-3576 \\ USA
}

\begin{abstract}
Minimum vertical clearances along highway systems impact the functionality of the road network. Extracting current routing clearances for each structure can be a challenging and hazardous task. Pavement changes and roadway rehabilitation projects can alter roadway geometry, complicating efforts to maintain accurate clearance databases. Inherently, traditional methods that are used to obtain the measurements routinely impede traffic flow and subject workers to dangerous environments. This study will examine the use of a Mobile LiDAR system and its applicability and accuracy to obtain vertical clearances on bridge structures. This study aims to propose an alternative method for extracting minimum clearances along highway systems while investigating the impact of work zone safety and traffic disruption. The proposed method is assessed using a statistical analysis of data obtained through traditional and Mobile LiDAR collections. Results show that there were no statistical differences between methods while the exposure to safety hazards was greatly reduced.
\end{abstract}

Keywords: LiDAR, MLS, Mobile LiDAR, bridge clearance, clearance database, oversized load, bridge heights.

\section{Introduction}

Obtaining accurate clearances from overhead structures provides essential information for the safe routing of oversized vehicles, especially along highly traveled routes such as interstates and parkways. According to the Bureau of Transportation Statistics (BTS, 2017) a total of 10,776 million tons of freight was transported using U.S. highways in 2015, with a projected growth to 14,829 million tons by 2045. The Federal Highway Administration (FHWA) has established design guidelines for overhead structures which accommodate for the safe travel of most vehicles. However, extracting current routing clearances for each structure can be a challenging and hazardous task. Pavement changes and roadway rehabilitation projects can alter roadway geometry, complicating efforts to maintain accurate clearance databases. Although many technologies have been successful in extracting vertical clearances, a recommended methodology has not been established. Current practices routinely involve disruptive lane closures, exposing workers and public motorists to dangerous environments.

\section{Current Methods}

Design guidelines for minimum vertical clearances have been established but extraction methods have not been specified. The decision has been left to state Department of Transportation (DOT) agencies to utilize the best method to obtain the necessary measurements. Although a variety of tools and techniques exist for acquiring minimum vertical clearances, a defined approach has not been determined. As new technologies are introduced, these methods continuously change. A review of previous and current techniques are outlined below.

2.1 Grade Rods. Most likely due to the simplistic approach, research for this method is sparse but mentioned as a previous extraction method and a current quality control technique. Normally used for surveying applications, grade rods are an extendable measurement device with graduated units used to determine differences in elevation. A study by Lauzon (2000), notes that vertical clearance extraction performed by ConnDOT utilized a fiberglass measurement rod to determine the minimum vertical clearances of overpasses. Measurements were taken from each lane line and recorded to overhead clearance diagrams. The study also notes that this method requires traffic protection, which can be substantial on interstates and in urban areas.

2.2 Total station. Total stations combine electronic EDM (Electronic Distance Measurement) technology and theodolites into a single unit. 
They digitally calculate and record horizontal directions, vertical directions and slope distances. Using an internal or external microprocessor these digital data observations can be adjusted and transformed to local X,Y,Z coordinates (US Army Corps of Engineers 2007).

A few key measurements are required when utilizing a total station to determine the height of overhead objects. The graphic in figure 1 illustrates the typical parameters needed to calculate object heights, also referred to as Remote Elevation Method (REM). The principle of the REM method is explained through figure 1, where a base point P1 is positioned vertically below point $\mathrm{P} 2$. From the instrument $\mathrm{P} 0$, a measurement is taken at the prism above base point $\mathrm{P} 1$, providing the slope distance d1. A subsequent measurement at P2 is used to calculate the angular difference $\alpha$, from P1. Given the height of the prism, the height (a) can then be calculated internally by the total station.

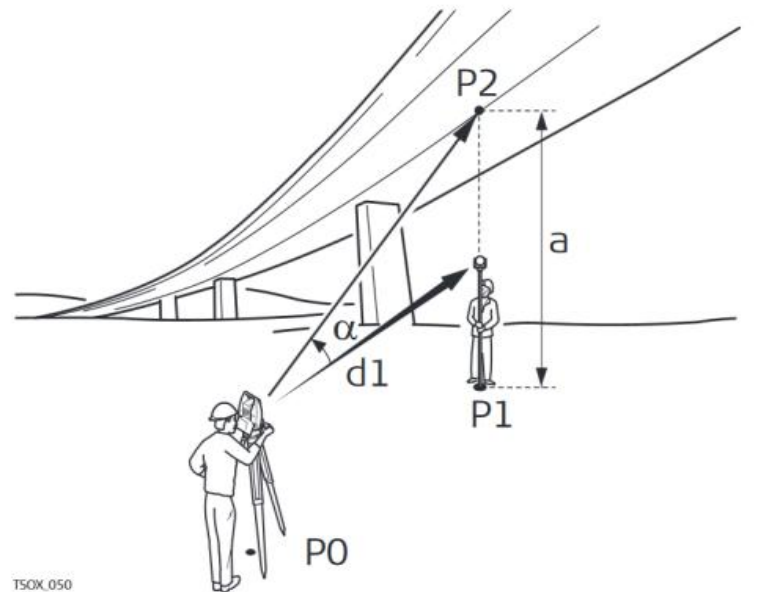

$\begin{array}{ll}\text { P0 } & \text { Instrument station } \\ \text { P1 } & \text { Base point } \\ \text { P2 } & \text { Remote point } \\ \text { d1 } & \text { Slope distance } \\ \text { a } & \text { Height difference from P1 to P2 } \\ \text { a } & \text { Vertical angle between base point } \\ & \text { and remote point }\end{array}$

Figure 1.Remote Elevation Method. Leica user Manual, 2014.

2.3 Laser Tape Measure. Techniques commonly used to aid in structural measurement include tape measurements combined with hand recording and optical methods (Banister, Raymond \& Baker, 1998). Since the publication of this study in 1998, advancements in distance measuring devices such as the laser tape measure have been introduced. Utilizing laser tape meters has proven to be a more efficient and simplified method to extract vertical clearances over analog methods. The device can be operated by a single individual providing quick and accurate measurements displayed in a digital format. Although an improvement over previous methods, extracting overpass clearances via laser tape is not without its challenges. Accurate readings are obtainable, though many aspects of height extraction are subject to the individual operator. Human error and judgement can adversely affect data quality. Visually assessing and determining the location of the lowest point overhead can be difficult, especially with changing roadway and structure geometry. Additionally, failure to hold a perpendicular angle to the roadway can result in inaccurate measurements.

2.4 Additional Technologies. Additional technologies have been developed; however, most are proprietary or developed privately. Little research is available and therefore was not included in this review.

\section{MLS technology}

3.1 Positioning components. Mobile LiDAR Systems (MLS) can be broken down into two sub-systems, comprised of geo-positioning and LiDAR components. The geopositioning system is composed of Global Navigation Satellite System (GNSS) receiver(s), a Digital Measurement Indicator (DMI), and an Inertial Measurement Unit (IMU). Georeferencing for MLS is determined by time variable position and orientation parameters. The three components of the geopositioning sub-system work together to synchronize outputs from the sensor(s). The GNSS antenna(s) collect satellite positioning data, the IMU records inertial measurements and orientation such as pitch, roll and yaw, while the DMI collects speed and linear distance information (Sokolova, Morrison \& Haakonsen, 2015). Post processing of data gathered by the positioning components yields an accurate representation of the vehicle's orientation parameters along the traveled route.

3.2 LiDAR components. The LiDAR system is made up of laser scanner(s), a control unit, a logging computer for data synchronization, and a laptop PC used to control system functions. Laser scanners measure surroundings using light pulses to obtain range and angle measurements. 


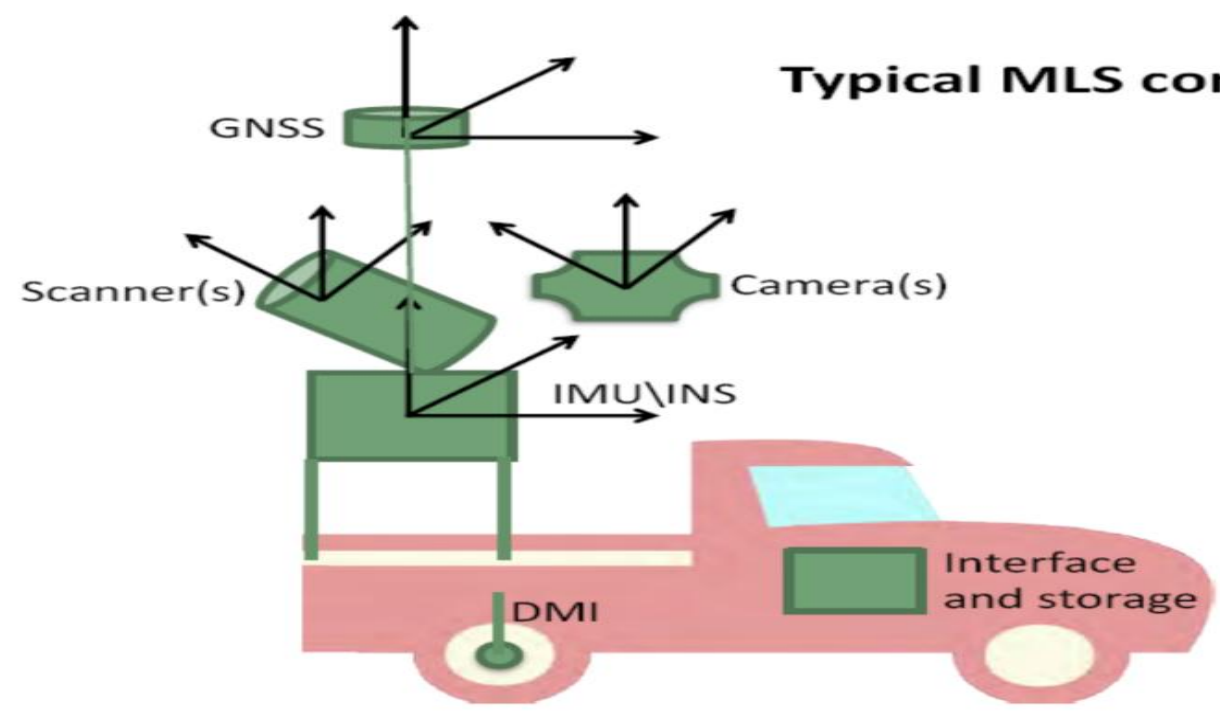

Figure 2.Typical MMS components. Retrieved from: Guidelines for the use of Mobile LiDAR in Transportation applications, 2013, p.55.

Figure 2 illustrates common components of an MLS system. Using Time-Of-Flight (TOF), the scanner sends a short laser pulse to the target, the time difference between the emitted and received pulses are used to determine the range from the scanner. The range $R$ can be calculated using the following expression: $R=1 /{ }_{2} c \Delta t$ Where $c$ is the speed of light and $\Delta t$ is the time of flight of the pulse (Puente et. al 2013).

\section{MLS Operations}

During operational procedures the geopositioning system collects synchronized location and orientation data from the GNSS antennas(s), IMU and DMI along with spatial data from the LiDAR sensors. Post processing navigational data produces a trajectory depicting a 3-dimensional representation of the traveled route. The generated trajectory is then used to synchronize LiDAR scanner outputs correlating to the time of incidence. The resulting data is an accurate 3D collection of surface measurements also referred to as a point cloud. In addition to spatial collection the LiDAR scanners(s) are also capable of extracting surface reflectance properties. Each scanned point can be assigned an intensity value based off of the return strength of the pulse. Varying surface properties affect the amplitude of the return pulses. Intensity values are assigned within a defined numeric range which can be displayed over graduated color tables. Intensity properties allow visualization software to differentiate between low reflectance surfaces such as asphalt and structures with highly reflective surfaces such as lane striping and signage. Figures 3 and 4 show the differences in point cloud data with and without intensity values, respectively.

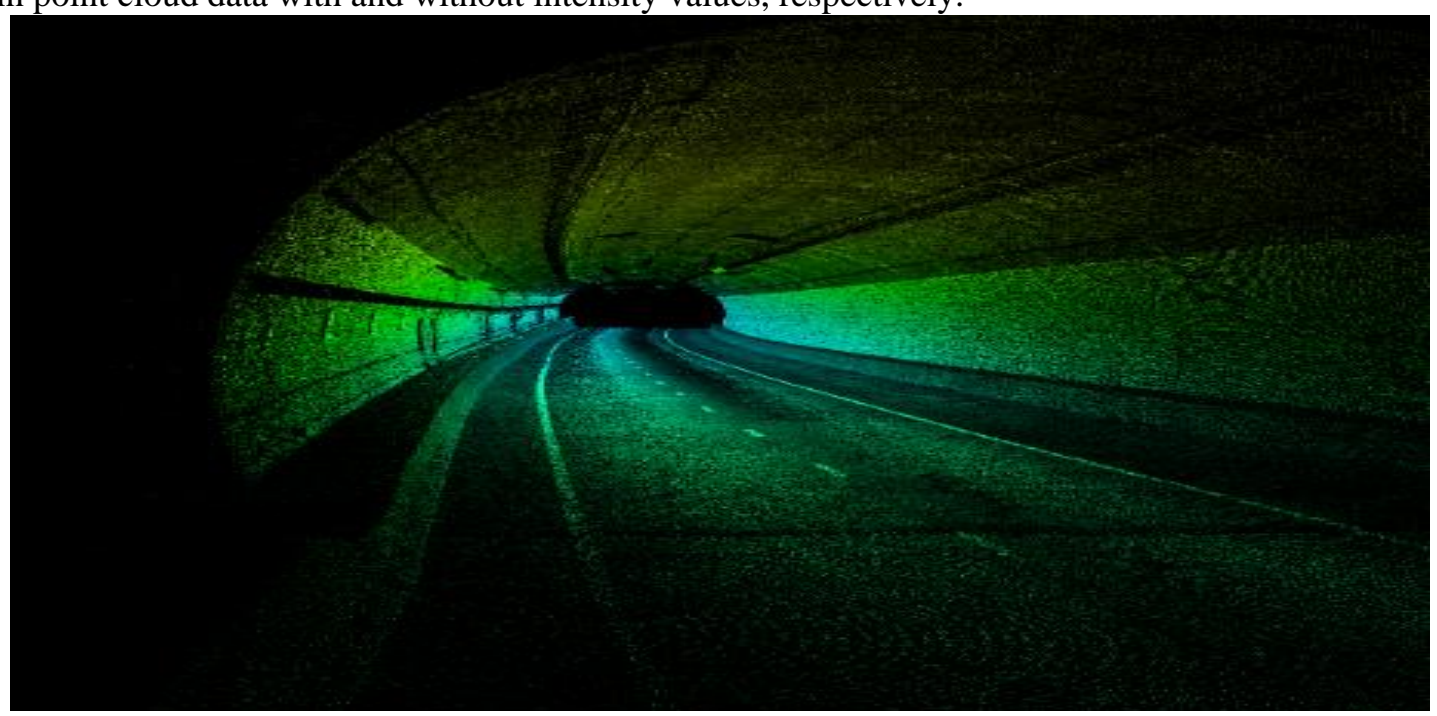

Figure 3. I-264 tunnel w/intensity values 


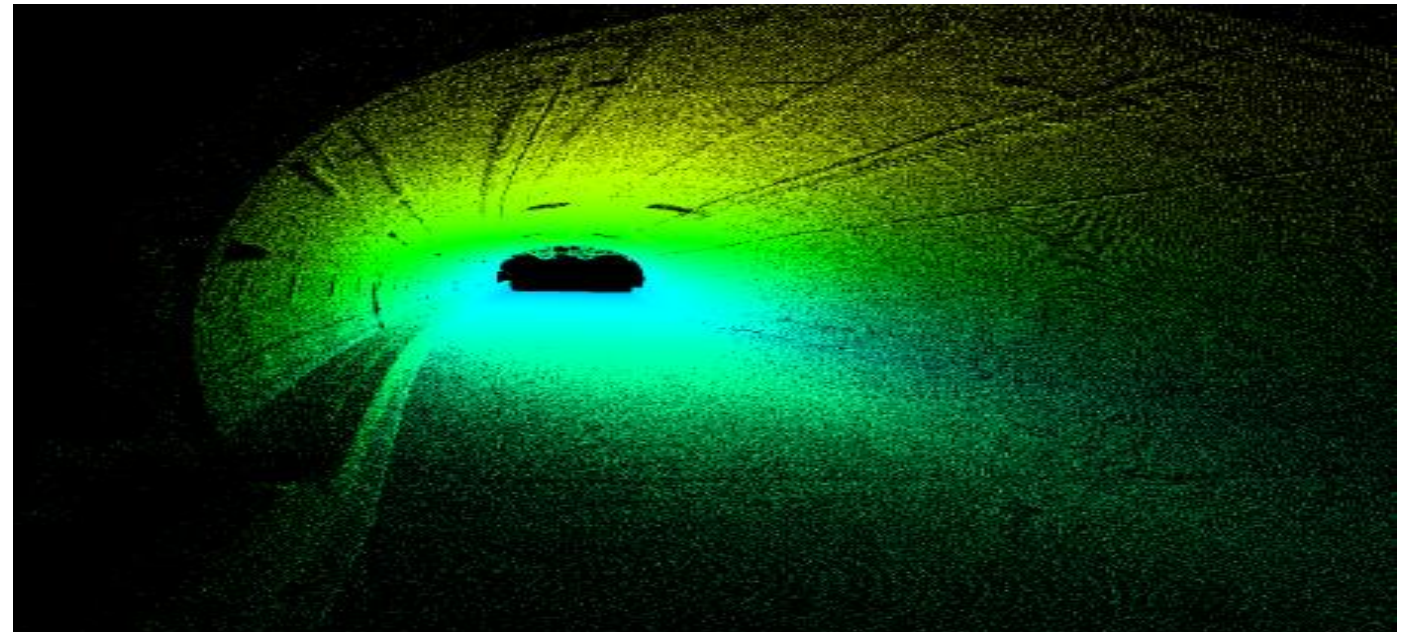

\section{Procedures}

Figure 4.I-264 tunnel w/o intensity values.

To compare differences between MLS and traditional methods the research identifies procedures and workflows currently used to extract vertical clearances from civil infrastructures. Three different locations were chosen for this study. MLS data was collected using an Optech Lynx Mobile Mapping System, utilizing two V100 laser scanners. Traditional extraction methods utilized a Spectre laser meter and a Nikon NPL-332 total station. Measurements extracted from the LiDAR data were processed using CAD software. Vertical clearances were extracted from each outside beam using roadway edge lines as a perpendicular reference. This study collected measurements obtained using each technique from the same location. Graphical representations of the data are analyzed along with a review of collection procedures and its perceived impact on traffic and safety.

The experimental procedures conducted are the same for each test area and are described as follows:

5.1 Laser Tape. The laser tape device was affixed to a survey rod and bipod to ensure level, perpendicular measurements from the roadway surface. Through the use of the integrated laser, the center of each beam was located and measured from roadway edge lines. In areas where no lane lines were present, a distance from 6" from the pavement edge was used. A PK nail was driven into the asphalt to ensure location accuracy between methods. The average of three measurements were taken from each location and recorded. The height of the laser tape affixed to the range pole was measured from the ground and added to each measurement to calculate total height.

5.2 Total Station. Using the PK nail as a reference, measurements from the total station were taken from the center of each exterior beam above the edge lane lines. The pole, bipod, and prism were placed under the targeted beam as shown in figure 5. The total station was set up and leveled. Using the REM method, measurements were collected and recorded for each test area.

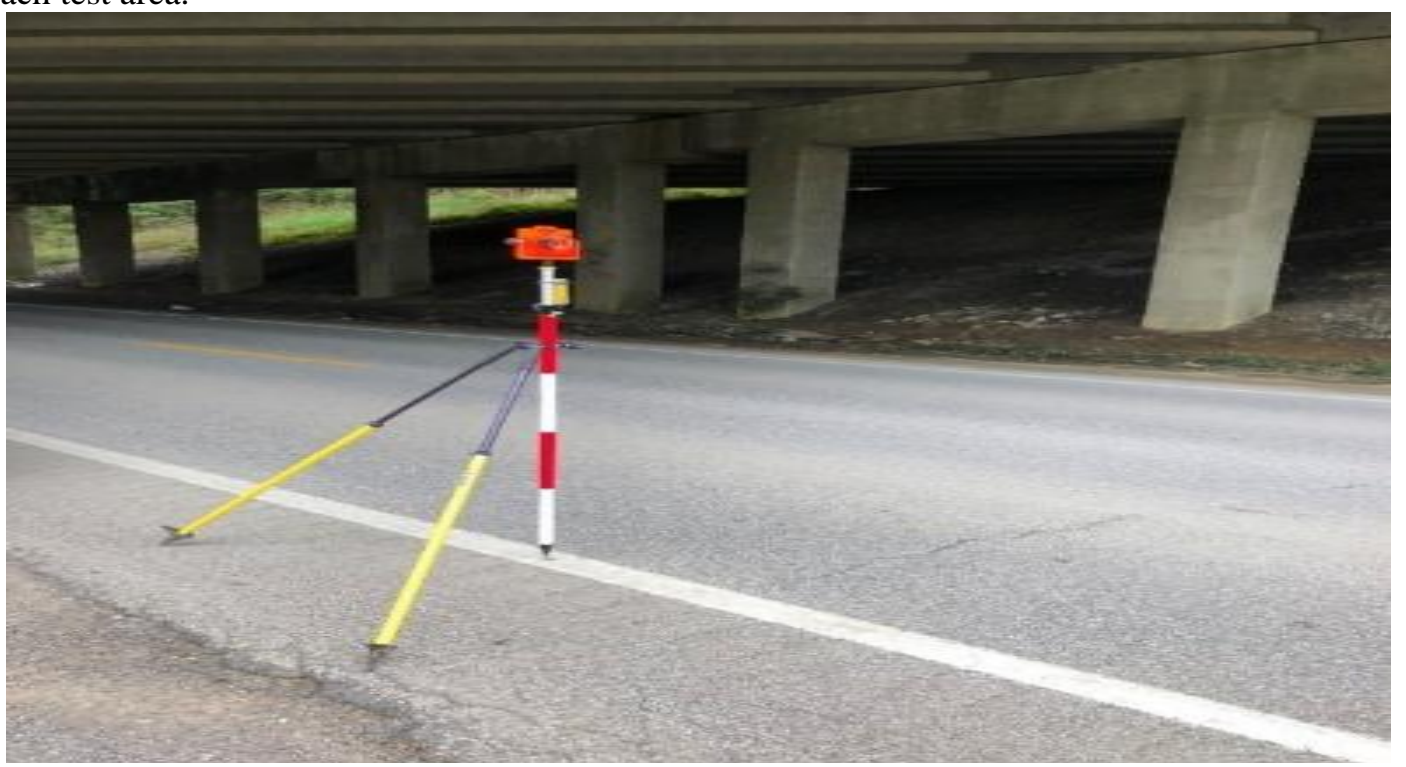

Figure 5. REM method setup. 
5.3 MLS. An Optech Lynx Mobile Mapping System as shown in figure6 was used to scan the project areas after the field measurements were completed. A boresight procedure as discussed below was performed in order to calibrate the system for the upcoming scan. Travelling at a constant speed of 40MPH, data was collected from each structure.

5.4 Post Processing. Trajectory and GPS information collected from the POS (Position Orientation System) during scanning operations was post processed through Applanix PosPac MMS software. Using data from continuously operating reference stations (CORS) to compute a set of corrections for the roving receiver, the software exports an accurate overall position and orientation solution. The exported smoothed best estimate of trajectory (SBET) file is GPS time stamped and used to align with correlational LiDAR outputs. Data used in this study was processed using six CORS stations, KYBO, KYTF, GRTN, KYTI, KYBU and KYTG as shown in figure 7. The corrected solution was exported using the Kentucky Single Zone coordinate system.

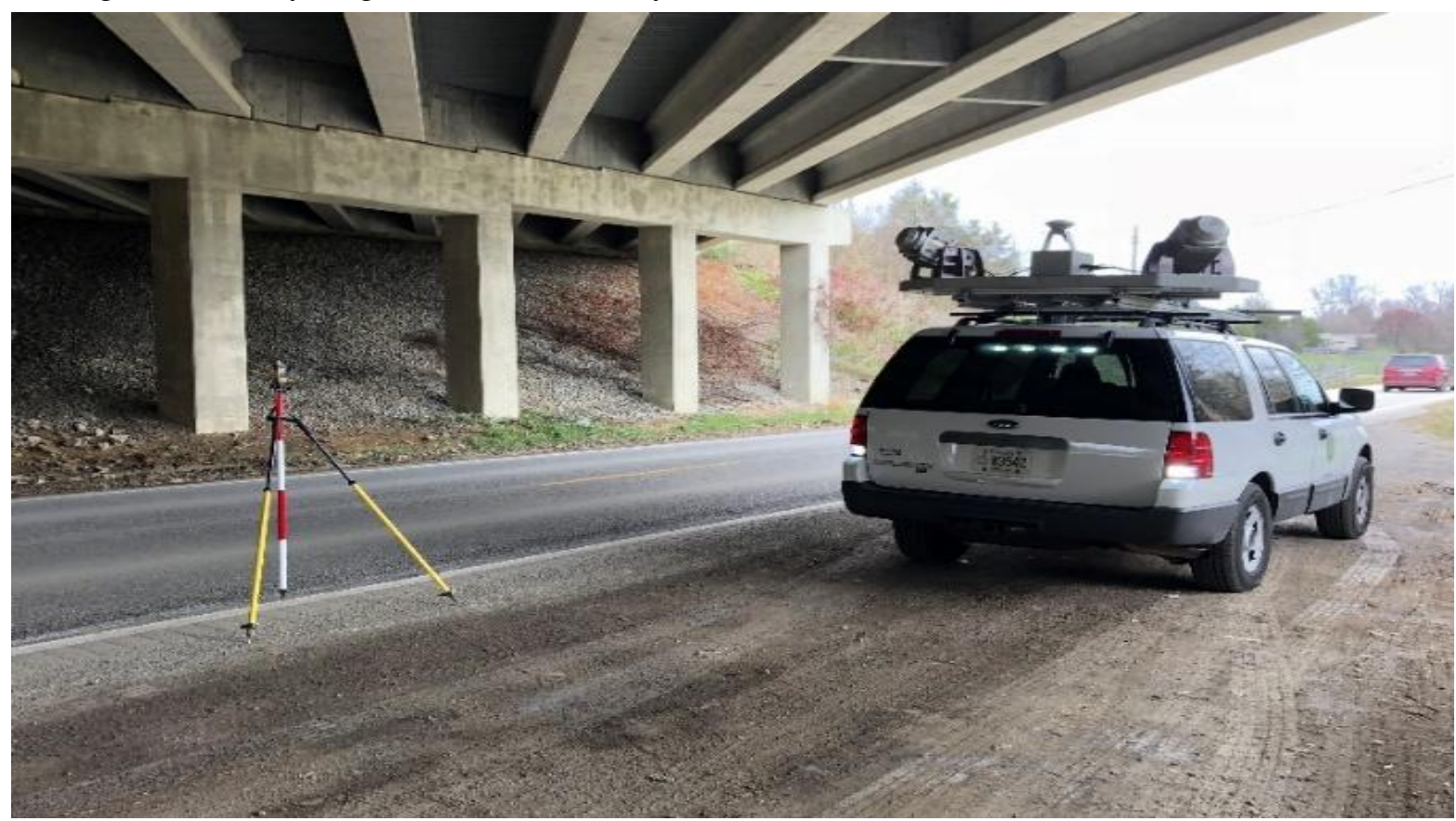

Figure 6. Optech Lynx MLS system.

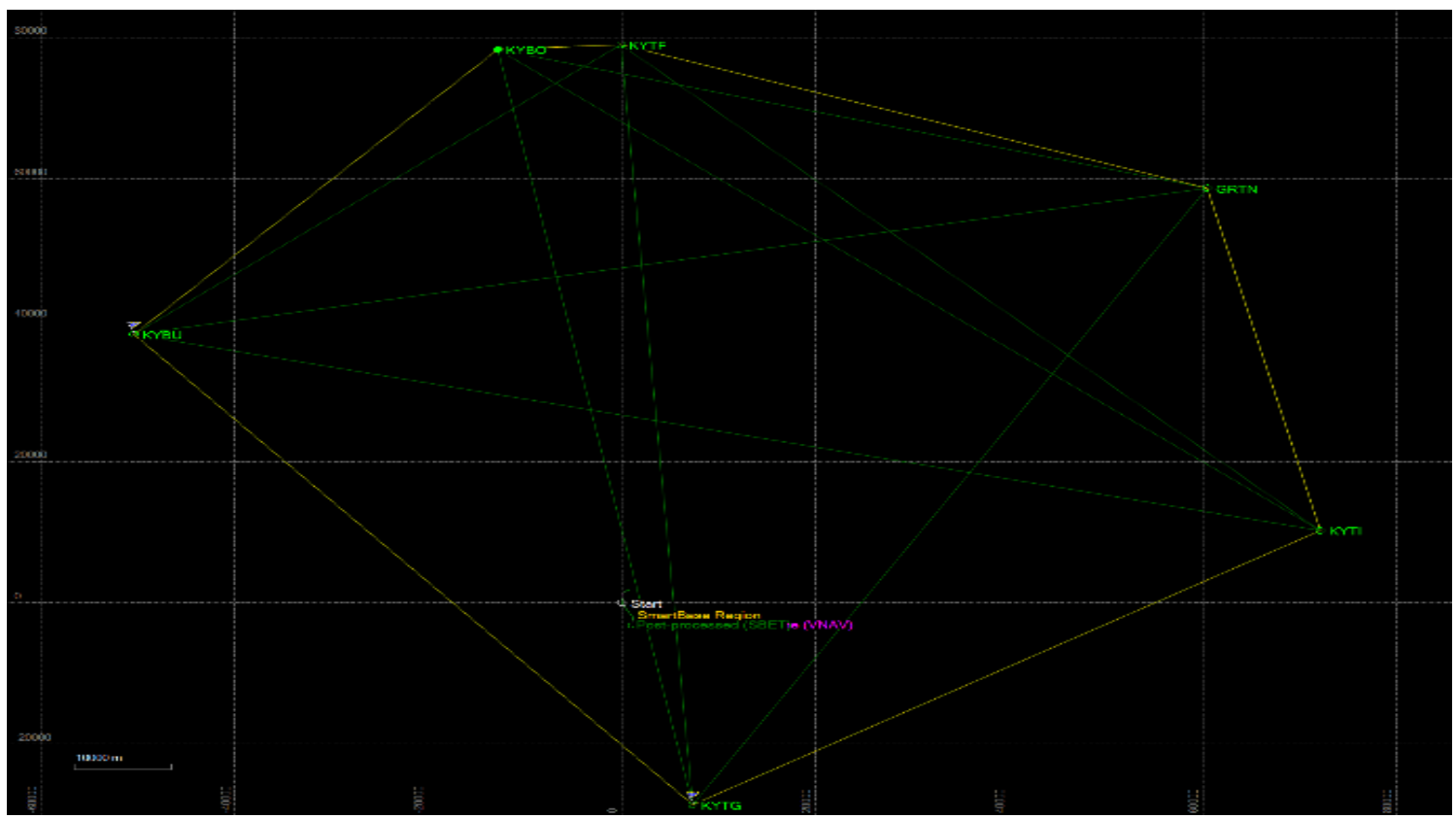

Figure 7. PosPac CORS Solution. 
5.5 System calibration/boresight. System calibration parameters were obtained using a boresight scan. Data was collected in order to calculate differences in like planar surfaces detected from each laser. Multiple passes produce a set of point clouds covering redundant surface observations. Recommended conditions for the boresight area include objects with multiple planar surfaces in various orientations. Areas with tall buildings or tree canopies should be avoided in order to retain consistent GPS data. During post-processing LMS software detects differences in planar surface orientations. Using the method of least squares, the software determines a set of unknown parameters from a set of redundant observations. Misalignments between the measurement axes of the IMU and laser scanners are calculated. The software applies a correctional value to offset errors in laser range, scan angle, sensor position and orientation. The values are then applied to the instrument in order to produce corrected data for the scan project (LMS for Lynx Users, 2013).

An urban intersection was chosen as the boresight location for this project. This area provided multiple buildings with limited overhead obstructions. Four passes were made in order to obtain planar surfaces from different orientations. Figure 8 shows planar surfaces extracted for boresighting calculations. Figure 9 shows the point cloud of the scanned area. Using the corrected parameters from the boresight, LMS software was used to export the point cloud for each overpass. Figure 10 shows an example of the exported point cloud for the Cincinnati Rd. location.

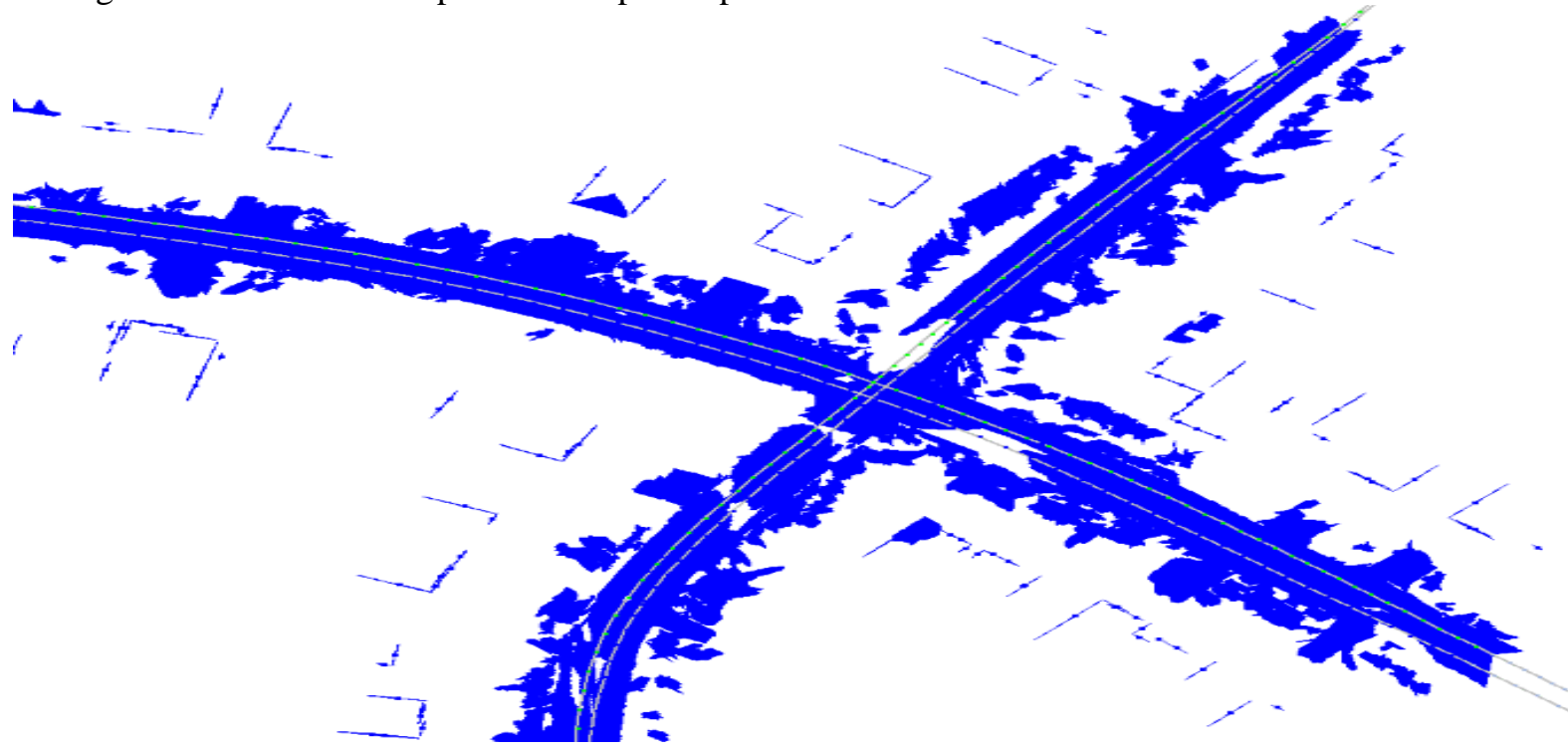

Figure 8. Boresight Planar Surfaces detection

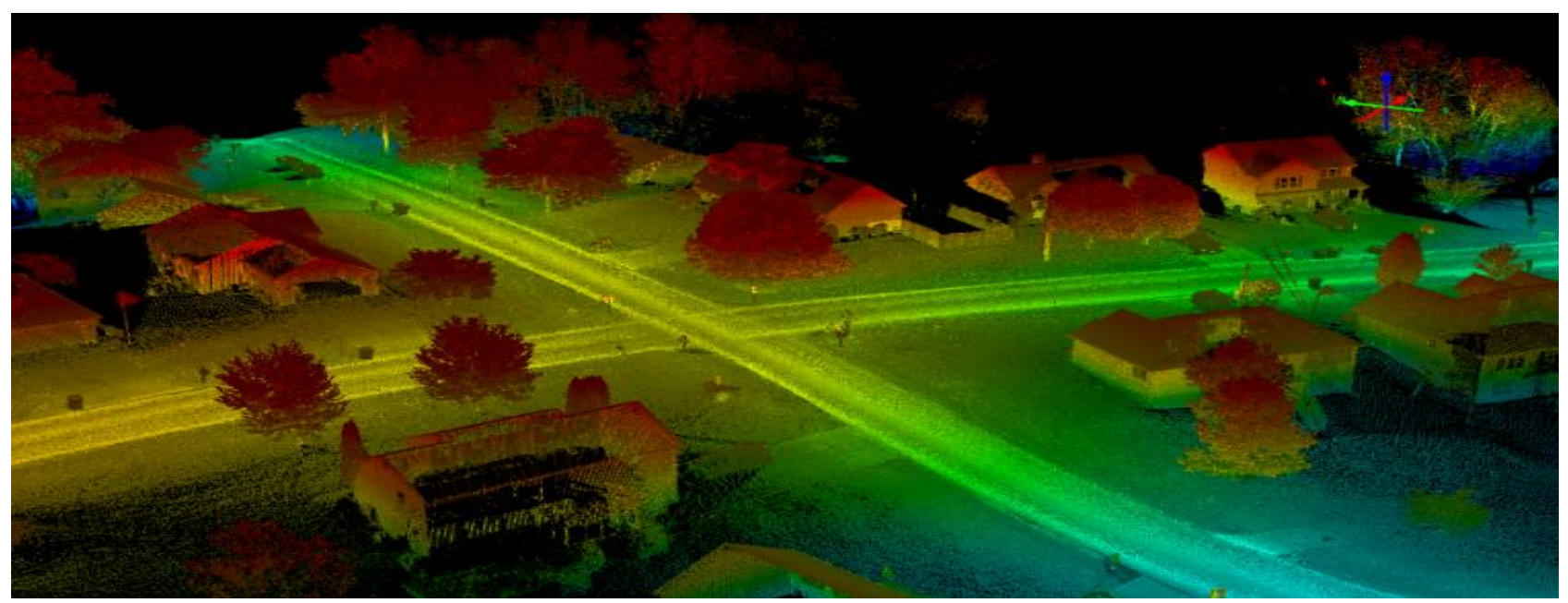

Figure 9. Boresight area point cloud. 


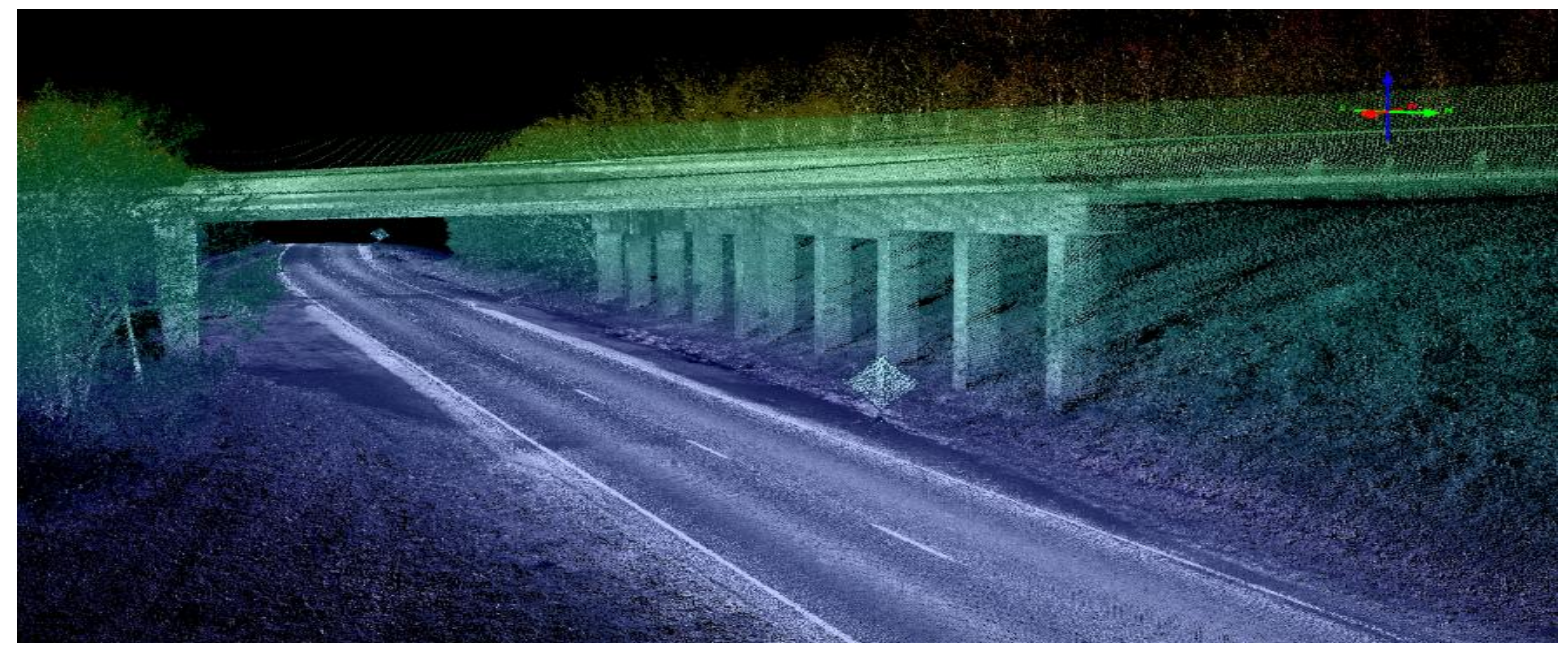

Figure 10. Cincinnati Rd point cloud

5.6 Clearance Extraction. A combination of Bentley Openroads Designer and TopoDOT software was used to extract clearances from the point clouds. Visualizing the data by intensity values, exterior lane lines were used as a reference path for the extraction tools. Given the referenced path, the software calculates the clearance values between the roadway and the overhead structure. Cross section examples for each project location depicting minimum clearances are shown in figures 11-13. Table 1 shows the clearance values for all measurement methods.

Table 1

Measurement Results (in US Survey Feet)

\begin{tabular}{|c|c|c|c|}
\hline \multicolumn{4}{|c|}{ Alexandria Dr. at New Circle Rd. } \\
\hline Location & LMS & Laser Tape & $R E M$ \\
\hline Southern Beam NB & 15.615 & 15.655 & 15.609 \\
\hline Northern Beam NB & 20.963 & 20.977 & 20.910 \\
\hline Southern Beam SB & 16.125 & 16.182 & 16.121 \\
\hline Northern Beam SB & 21.393 & 21.412 & 21.317 \\
\hline \multicolumn{4}{|l|}{ Cincinnati Rd at I-75 } \\
\hline Location & LMS & Laser Tape & $R E M$ \\
\hline Southern Beam NB & 17.626 & 17.646 & 17.588 \\
\hline Northern Beam NB & 21.877 & 21.888 & 21.810 \\
\hline Southern Beam SB & 16.701 & 16.723 & 16.664 \\
\hline Northern Beam SB & 20.824 & 20.837 & 20.789 \\
\hline \multicolumn{4}{|l|}{ Rogers Gap Rd at I-75 } \\
\hline Location & LMS & Laser Tape & $R E M$ \\
\hline Southern Beam NB & 16.856 & 16.844 & 16.861 \\
\hline Northern Beam NB & 15.938 & 15.957 & 15.906 \\
\hline Southern Beam SB & 16.405 & 16.439 & 16.431 \\
\hline Northern Beam SB & 15.679 & 15.693 & 15.691 \\
\hline
\end{tabular}


Figure 11.Alexandria Rd. minimum clearances.
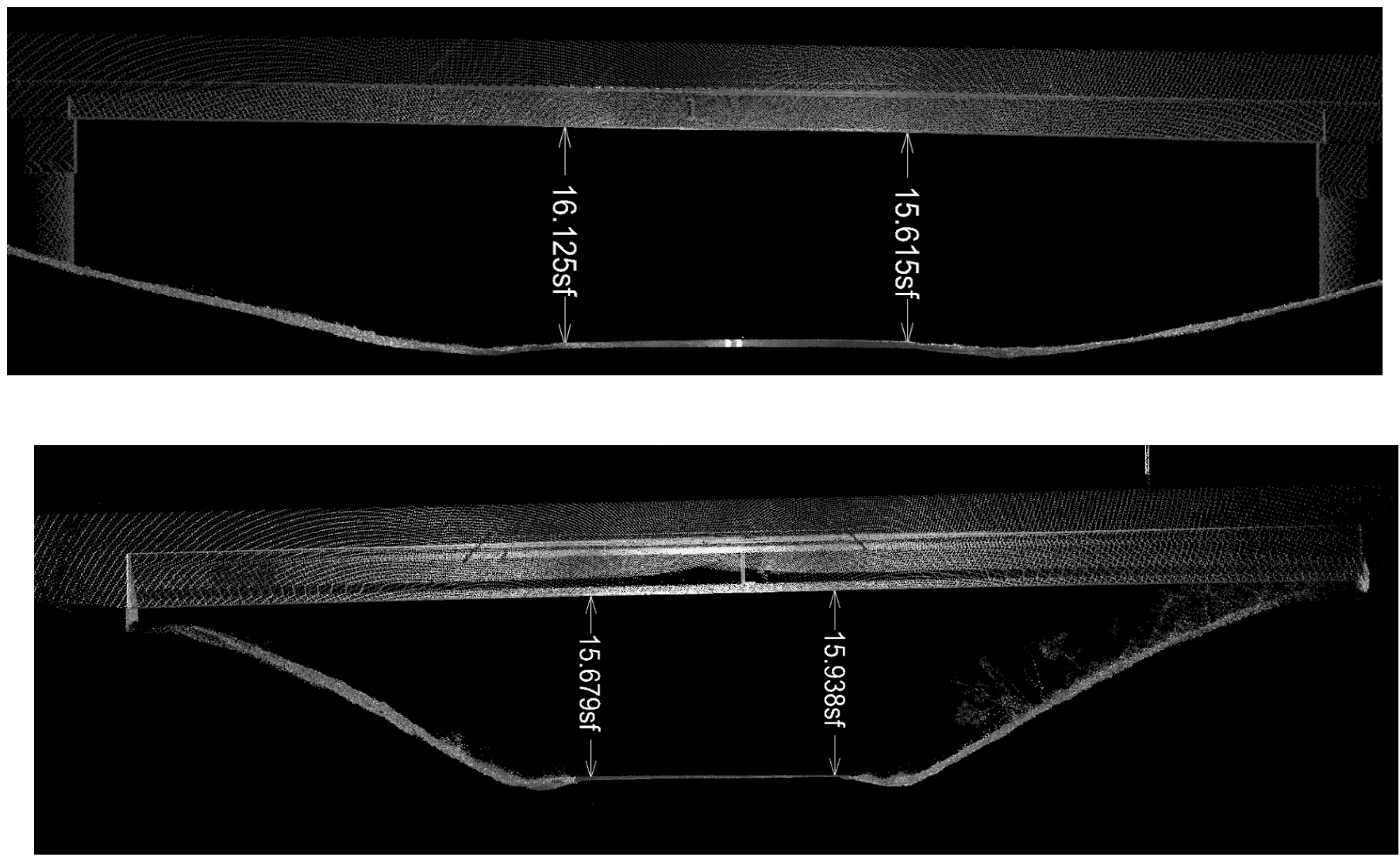

Figure 12.Cincinnati Rd. minimum clearances.

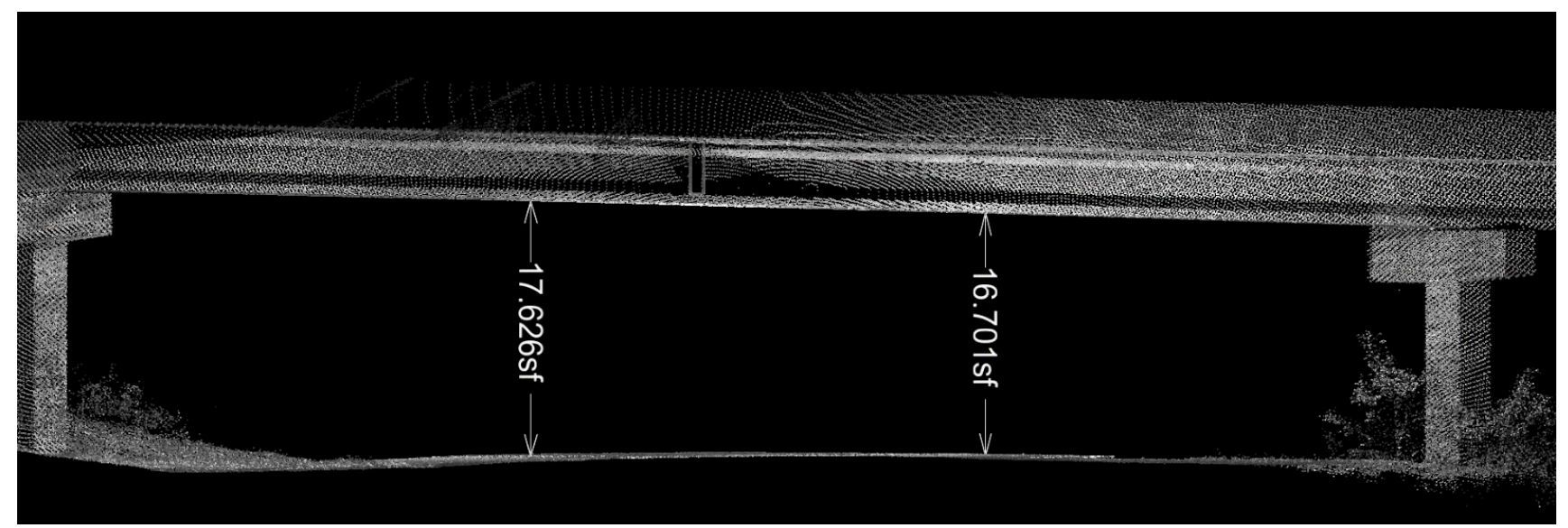

Figure13. Rogers Gap Rd. minimum clearances

\section{Method of Data Analysis}

The results of this study can be divided into two parts. First a One-Way Anova test was run to statistically show how each method of clearance extraction performed relative to the others under the same conditions. The researcher tested the hypothesis that the Laser tape, REM, and LiDAR methods did not show statistical differences between each collected dataset using an $\alpha=0.05$. Table 2 provides a summary of the defined alternative hypotheses $\left(\mathrm{H}_{1}\right)$ as well as the null hypotheses $\left(\mathrm{H}_{0}\right)$. The null hypothesis $\left(\mathrm{H}_{0}\right)$ will be rejected if the one-way ANOVA test falls into the rejection region $(\mathrm{p}<.5)$. Otherwise, the null hypothesis would be retained. The laser tape group was represented by $\mu_{1}$, REM method by $\mu_{2}$, and LiDAR by $\mu_{3}$. Sample size for each group is represented by $n$. Second, the researcher compared each method on operational procedures, impact on work zone safety and traffic disruption. 


Table 2
Hypothesis Summary
$\mathrm{H}_{0}: \mu_{1}=\mu_{2}=\mu_{3}$
$\mathrm{H}_{1}:$ Means are not all equal

\section{Results and Discussion}

A one-way ANOVA was conducted to determine if collected measurements were statistically different for groups using different collection methods. Methods were classified into three groups: Laser tape $(n=12)$, Mobile LiDAR $(n=12)$, and REM $(n=12)$. The mean of measurement increased from the REM $(n=12, M=17.958, S D=2.433)$, to Mobile LiDAR $(n=12, M=18.00, S D=2.481)$, to Laser Tape $(n=12, M=18.021, S D=2.477)$ measurement method groups, in that order, but the differences between these groups was not statistically significant, $F(2,33)=0.002, p=.998$. The group means were not statistically significant different ( $p>.05)$. Therefore, we cannot reject the null hypothesis and we cannot accept the alternative hypothesis.

The second analysis looks to discuss each methods impact on safety, traffic, and operational procedures. Due to safety concerns, site selection for this project was based on areas with low traffic flow. However, the procedures undertaken can be theoretically applied to high traffic areas.

7.1 Remote Elevation Method. Data collected using the REM method proved to be the most challenging. The magnified sights of the total station limited the field of view, dark conditions and similar materials made it difficult to distinguish features and locate individual beam centers. Small changes in vertical inclination of the instrument can significantly change the result of the intended measurement, this effect was apparent when performing operations at close range. Setup and data acquisition time was moderate, multiple setups for each structure were required in order to retain a line of sight to the intended target. Multiple setups introduce a greater possibility for error. Improper leveling and varying instrument heights can skew results. This method requires workers to occupy areas of interest below the structure, potentially exposing them to hazardous situations. Depending on site layout and bridge design this method may require the use of traffic control measures to provide a safe working environment.

7.2 Laser Tape. The laser tape proved to be the fastest method of acquiring vertical clearance data when a limited number of data points are needed. Results are obtained in real time and the operation can be carried out by a single individual. This method directly subjects workers to vehicular traffic hazards for the longest duration. Individuals must level the instrument under the overhead target when acquiring measurements, potentially distracting them from surrounding hazards. The Laser tape, when added to a bipod and prism pole can mitigate human error when used properly. However, if the instrument is out of level the correct value will not be obtained. Depending on site conditions, this method may require additional traffic control measures.

7.3 MLS. Results of the analysis show the MLS dataset are highly correlational to the laser tape and REM methods. This method did not require any workers to be present under the structures and had little to no impact on traffic. Traffic control measures are usually not required unless needed under special circumstances. Unlike the other two methods, LiDAR technology does not provide real time data. Additional time is needed to post process the data and extract the results. Boresighting procedures are also recommended before data collection. Unforeseen complications in GPS signal quality or hardware components can negatively impact accuracies. LiDAR systems require additional resources such as hardware, software, and trained personnel and therefore may not always be a feasible option. Data collected for this study concentrated on extracting clearance heights from exterior lane lines. However, super elevated or crowned roadway geometry can result in overhead clearance variability, reducing minimum clearances in other locations below the structure. In these scenarios, additional traffic control measures may be needed to properly access and measure these areas when using the REM or laser tape methods.

\section{Conclusion}

No significant statistical differences were observed between the laser tape, REM, and MLS methods. Therefore, the study found that all three methods of vertical clearance extraction are capable of providing accurate measurements under the right conditions. The selection of which technology to utilize involves multiple factors such as time, site conditions, environmental conditions, resource availability, and safety concerns. The laser tape and REM methods provide a proven option to extract clearances on small scale projects in low traffic areas where safety concerns can be adequately managed. Mobile LiDAR is best suited for projects involving multiple structures, variable overhead clearances, hazardous conditions or areas prone to traffic congestion such as interstates or parkways. 


\section{Future Research}

While the current research provides a solid basis for the use of MLS systems to determine minimum clearances for bridge structures, the technology is not a one size fits all approach. This study identifies several key points, however, a cost benefit analysis encompassing all aspects of various projects and methods would further aid in future decisionmaking processes.

\section{References}

Bannister, A., Raymond, S., and Baker, R. (1998). Surveying. Addison- Wesley, New York.

Bureau of Transportation Statistics (2007). Freight Facts and Figures. [Online] Available: https://www.bts.gov/sites/bts.dot.gov/files/docs/FFF_2017.pdf

Lauzon, R. G. (2000). Automated vertical clearance measurement during photolog operations. ConnDOT, Report No. 2220-F-2000-4. [Online] Available: https://rosap.ntl.bts.gov/view/dot/14168

LMS for Lynx User Manual (2013). Optech Corp, Vaughan, ON Canada. (Chapter 5)

Olsen, M. J., Roe, G. V, Glennie, C., Persi, F., Consulting, P., Reedy, M, Tuss, H. (2013). NCHRP Guidelines for the use of Mobile Lidar in Transportation Applications. (15)44, 7-14.

Puente, I., González-Jorge, H., Martínez-Sánchez, J., \& Arias, P. (2013). Review of mobile mapping and surveying technologies. Measurement: Journal of the International Measurement Confederation, 46(7), 2127-2145. [Online] Available: http://doi.org/10.1016/j.measurement.2013.03.006

Sokolova, N., Morrison, A., \& Haakonsen, T. A. (2015). Public road infrastructure inventory in degraded global navigation satellite system signal environments. Journal of Geodetic Science, 5(1), 17-18. [Online] Available:http://doi.org/10.1515/jogs-2015-0002

US Army Corps of Engineers (2007). Engineering and design control and topographic surveying, The Army Corps of Engineers. [Online] Available: http://publications.usace.army.mil/publications/eng-manuals/EM 